Historic, Archive Document

Do not assume content reflects current scientific knowledge, policies, or practices. 
62,23

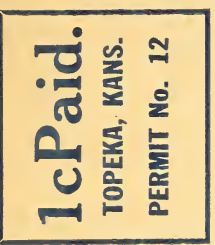

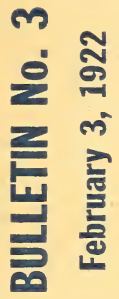

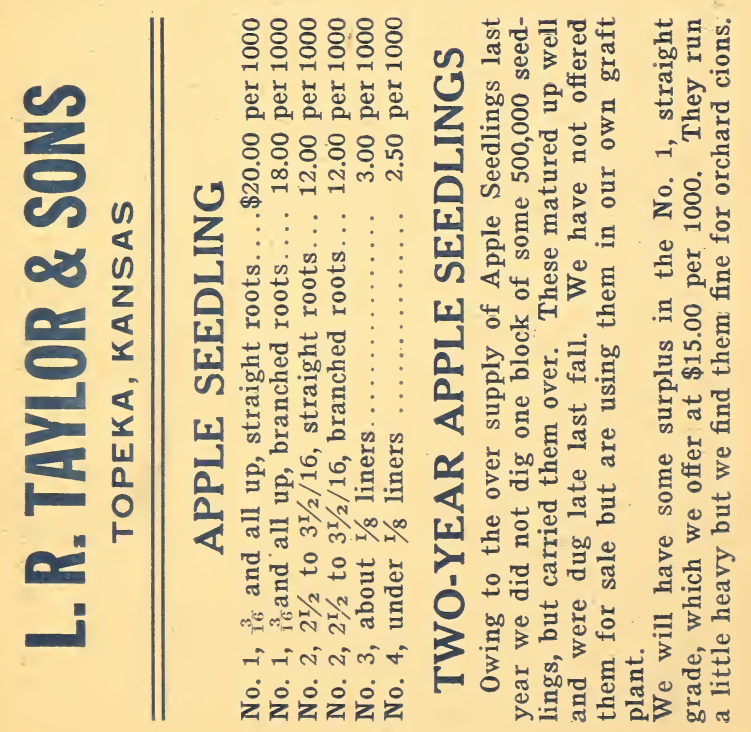




\section{ONE-YEAR PEACH}

Some of the items below have been sold but we still have a good supply in all grades.

We have a large surplus of Champions and will furnish these at 25 per cent off the quoted price.

Under

$1 \frac{9}{16} \quad \frac{7}{16} \quad \frac{5}{16} \quad \frac{5}{16}$

15 c 15 c 12 c 10 c $8 c$

Alexander .......... $20 \quad 72 \quad 20 \quad 20 \quad 75$

Belle of Georgia ..... $480 \quad 470 \quad 170 \quad 100 \quad 50$

Capt. Ede ......... $320 \quad 440 \quad 566 \quad 540 \quad 590$

Champion ......... $1463 \quad 18702000 \quad 5000 \quad 4000$

Chinese Cling $\ldots \ldots \ldots . \quad 170 \quad 87 \quad 432 \quad 248 \quad 551$

Carman .......... $882 \quad 1224 \quad 1000 \quad 1524 \quad 1149$

Crosby ............ $130 \quad 160 \quad 160 \quad 92 \quad 75$

Elberta .......... $500 \quad 800 \quad 700 \quad 400 \quad 300$

Early Crawford ...... $1035 \quad 1300 \quad 2000 \quad 2625 \quad 2500$

Fitzgerald $\ldots \ldots \ldots \ldots .100 \quad 200 \quad 750 \quad 1000 \quad 1000$

Foster ............ $250 \quad 600 \quad 1000 \quad 800 \quad 650$

Heath Cling ....... $450 \quad 700 \quad 50 \quad 250 \quad 150$

Late Crawford ...... $300 \quad 450 \quad 1000 \quad 700 \quad 500$

Matthews Beauty ... $300 \quad 250 \quad 1000 \quad 500 \quad 450$

Mayflower ......... $400 \quad 400 \quad 200 \quad 200 \quad 50$

Mamie Ross ....... $500 \quad 350 \quad 150 \quad 50 \quad 100$

Mt. Rose ......... $200 \quad 300 \quad 300 \quad 250 \quad 400$

0. M. Cling ........ $950 \quad 500 \quad 250 \quad 100 \quad 50$

O. M. Free ......... $250 \quad 450 \quad 950 \quad 1500 \quad 2000$

Salway ........... $150 \quad 900 \quad 1000 \quad 700 \quad 500$

Smock .......... $50 \quad 550 \quad 90 \quad 550 \quad 300$

Stump ........... 680 $680^{-} 300 \quad 750 \quad 390 \quad 450$

Wonderful .......... $300 \quad 140 \quad 150 \quad 30 \quad 235$

Greensboro ........ $20 \quad 60 \quad 170 \quad 160 \quad 150$

ONE-YEAR PLUM Under

$1 \frac{1}{16} \quad \frac{9}{16} \quad \frac{7}{16} \quad \frac{5}{16} \quad \frac{5}{16}$

30 c 25c 20c 15c 10 c

\begin{tabular}{|c|c|c|c|c|c|}
\hline Abundance & 180 & 60 & 70 & 60 & 20 \\
\hline Burbank ............ & 260 & 300 & 660 & 200 & 25 \\
\hline DeSoto & . & 15 & 80 & 30 & 3 \\
\hline Forest Garden ....... & 20 & 35 & 270 & 160 & 13 \\
\hline 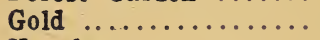 & 180 & 140 & 150 & 40 & 10 \\
\hline Hanska ............ & - & 75 & 400 & 250 & \\
\hline Hawkeye $\ldots \ldots \ldots$. & 10 & 45 & 240 & 100 & \\
\hline 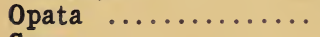 & 600 & 330 & 700 & 180 & \\
\hline$\ldots \ldots \ldots \ldots$ & $\cdots$ & 60 & 460 & 300 & प्र \\
\hline$\ldots \ldots \ldots \ldots \ldots$ & 170 & 156 & 330 & 100 & 12 \\
\hline Wickson $\ldots \ldots \ldots \ldots$ & 30 & 60 & 140 & 50 & \\
\hline Goose ......... & 550 & 130 & 220 & 100 & 10 \\
\hline$\ldots \ldots \ldots \ldots$ & ... & 28 & 200 & 180 & \\
\hline 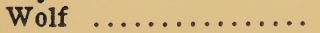 & & 40 & 150 & 60 & \\
\hline
\end{tabular}




\section{ONE-YEAR APRICOT}

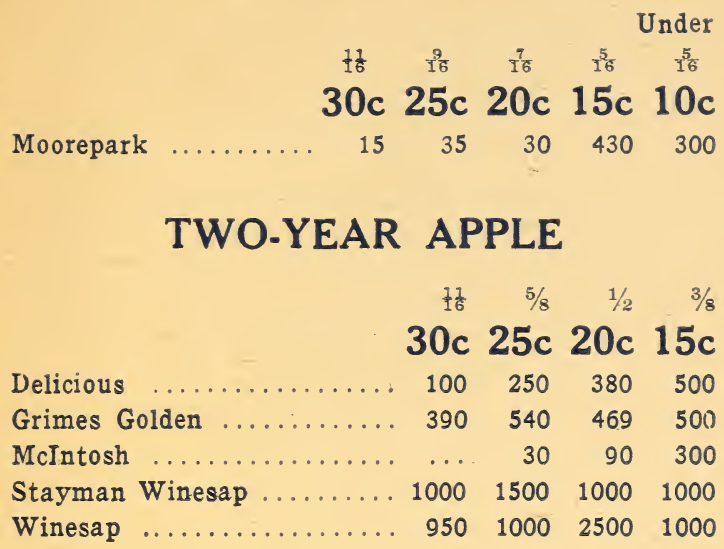

\section{ONE- and TWO-YEAR PEAR}

\begin{tabular}{|c|c|c|c|c|c|c|}
\hline & \multicolumn{5}{|c|}{ Under } \\
\hline & & $\frac{11}{6}$ & $\frac{9}{16}$ & $1 / 2$ & $2-3$ & $2-3$ \\
\hline & & $30 c$ & $25 c$ & $20 c$ & $15 c$ & $10 c$ \\
\hline Kieffer & & 2500 & 800 & 200 & 1000 & 200 \\
\hline Anjou & $\ldots \ldots \ldots \ldots \ldots$ & $\ldots$ & $\ldots$ & 290 & 350 & 50 \\
\hline Bartlett & $=\ldots \ldots \ldots \ldots$ & $\ldots$ & ... & $\ldots$ & 160 & 140 \\
\hline Clapp's & $\ldots \ldots \ldots \ldots \ldots$ & $\ldots$ & $\ldots$ & 220 & 280 & 90 \\
\hline Duchess & $\ldots \ldots$ & $\ldots$ & $\ldots$ & 220 & 180 & 90 \\
\hline Garber & & 130 & 170 & 170 & 150 & $\ldots$ \\
\hline Seckel & $\ldots \ldots \ldots \ldots \ldots$ & $\ldots$ & $\ldots$ & 190 & 220 & 90 \\
\hline
\end{tabular}

\section{TWO-YEAR CHERRY}

$60 \mathrm{c} 50 \mathrm{c} 35 \mathrm{c} 20 \mathrm{c}$

Early Richmond ......... $100 \quad 150 \quad 200 \quad 150$ Montmorency ............ $200 \quad 400 \quad 350 \quad 150$

\section{GRAPE}

Concord, 1 year, No. 1 (Michigan grown) $\$ 4.00$ per 100

\section{JAPAN PEAR SEEDLINGS}

No. $1, \frac{3}{16}$ and up .............. $\$ 15.00$ per 1000

No. $2,2 / 16$ to $\frac{3}{16} \ldots \ldots \ldots \ldots \ldots \ldots . \ldots 12.00$ per 1000 


\section{FRENCH PEAR SEEDLING}

No. $1, \frac{3}{16}$ and all up $\ldots \ldots \ldots \ldots . . . \ldots$. . . . . . .

No. $2,2 / 16$ to ${ }_{16}^{3} \ldots \ldots \ldots \ldots \ldots \ldots \ldots \ldots$. . . . . . . . . .

No. 3, about $2 / 16 \ldots \ldots \ldots \ldots \ldots \ldots . . . \ldots 10.00$ per 1000

No. 4, for lining out............ 8.00 per 1000

\section{APPLE GRAFTS}

Whole Root, 6 in. cion, 15 M and up. . $\$ 15.00$ per 1000

Whole Root, $\sigma$ in. cion, 5 to 15 M ... 20.00 per 1000

Whole Root, 6 in. cion, 1 to 5 M..... 22.00 per 1000

Piece Root, 4 in. root, 6 in. cion, $15 \mathrm{M}$

and up ................... 10.00 per 1000

Piece Root, 4 in. root, 6 in. cion, 5 to

$15 \mathrm{MI} \ldots \ldots \ldots \ldots \ldots \ldots \ldots \ldots \ldots \ldots$. 12.00 per 1000

Piece Root, 4 in. root, 6 in. cion, 1 to

$5 \mathrm{M}$

14.00 per 1000

\section{SHADE TREES}

Elm, American White, 3-3 $\mathrm{x} / 2$ inches......each, $\$ 3.00$

Elm, American White, $2 \mathrm{~T} / 2-3$ inches.......each, 1.50

Elm, American White, $2-2 \mathrm{~T} / 2$ inches.......each 1.00

Elm, American White, $2-2^{1} / 2$ inches......each, 1.00

Elm, American White, $1 \mathrm{x} / 2-2$ inches.......each, .60

Elm, American White, $11 / 4-11 / 2$ inches.... each, .40

Elm, American White, $1-1 \frac{1}{4}$ inches......each, .25

Elm, American White, $6-8 \mathrm{ft} . . . \ldots . . .$. each, .20

Maple, Silver, $2-2 \mathrm{r} / 2$ inches............each, $\quad .80$

Maple, Silver, $1 \mathrm{r} / 2-2$ inches..............each, .50

Maple, Silver, $11 / 4-11 / 2$ inches...........each, .35

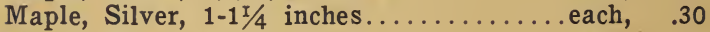

Maple Silver, $6=8 \mathrm{ft} . \ldots \ldots \ldots \ldots \ldots \ldots$ each, $\quad .20$

Catalpa (same price as Maple).

Stock quoted in this list is covered by Entomologist's Certificate. All stock is in cellar except Shades, and can be shipped promptly.

Prices are net; boxing extra, at cost.

\section{APPLE CIONS}

We need a few varieties of Apple Cions, mostly early sorts. Send us list of surplus with prices. Would like to exchange peach trees or No. 3 and No. 4 Apple Seedlings for Cions as far as possible.

L. R. TAYLOR \& SONS, Topeka, Kansas. 